\title{
Prevalencia y factores asociados al síndrome de fragilidad en adultos mayores en la consulta de atención primaria de EsSalud, enero-abril 2015. Chiclayo, Perú
}

\author{
Milton Carlos Gonzáles-Mechán ${ }^{1, a}$, Juan Leguía-Cerna ${ }^{1,2, a}$, Cristian Díaz-Vélez ${ }^{1,2, b}$
}

\section{RESUMEN}

Objetivos: identificar la prevalencia y factores asociados al síndrome de fragilidad en adultos mayores en la consulta de atención primaria de EsSalud, Chiclayo-Perú.

Materiales y métodos: Se realizó un estudio descriptivo, prospectivo y transversal, cuya muestra fue de 326 adultos mayores pertenecientes a la zona urbana y distritos periféricos de Chiclayo que acuden a la consulta externa en los policlínicos Naylamp y Chiclayo Oeste. Se les aplicó una ficha de recolección de datos que incluía variables sociodemográficas, presencia de comorbilidad y polifarmacia, y los criterios clínicos del síndrome de fragilidad.

Resultados: La prevalencia de fragilidad fue de $17.5 \%$ y la de pre-fragilidad, $40.9 \%$. Los criterios clínicos de fragilidad más frecuentes fueron: cansancio auto referido (42.3\%) y fuerza de prensión disminuida (32.8\%). En el análisis bivariado, se encontró asociación con edad, grado de instrucción, ocupación (clase IV y V, referente a trabajos manuales), comorbilidad (clase II, enfermedad asintomática o enfermedad asintomática que requiere medicación pero que está bajo control), polifarmacia, anemia, enfermedad de Parkinson y enfermedad neurológica no vascular. El modelo predictor final, que incluyó la edad $(1,08$, IC95\%: 1,03-1,12), grado de instrucción superior como variable preventiva $(0,21$, IC $95 \%$ : 0,07$0,62)$, comorbilidad tipo II $(11,08$, IC95\%: $1,45-84,38)$ y polifarmacia $(2,49$, IC95\%: $1,24-5,03)$, predice la probabilidad de tener síndrome de fragilidad en un $75,6 \%$.

Conclusiones: Existe una alta prevalencia de fragilidad en la población de adultos mayores que acuden a la consulta de atención primaria y una asociación significativa con la edad, grado de instrucción superior, comorbilidad tipo II y polifarmacia.

Palabras clave: Salud del adulto; salud del anciano; anciano frágil; atención primaria de salud (Fuente: DeSC BIREME).

\section{Prevalence and factors associated with fragility syndrome in older adults attending the EsSalud primary health care service, January - April 2015. Chiclayo, Peru}

\begin{abstract}
Objectives: To identify the prevalence and factors associated with fragility syndrome in older adults attending the EsSalud (Peruvian Social Security Health Insurance) primary health care service, Chiclayo - Peru.

Materials and methods: A descriptive, prospective and cross-sectional study was conducted on a sample consisting of 326 older adults from urban areas and surrounding districts of Chiclayo, who attend the outpatient service at the Hospital Naylamp and Policlínico Chiclayo Oeste. A data collection sheet including social-demographic variables, comorbidity and polypharmacy, and fragility syndrome clinical criteria was filled in.

Results: The prevalence of fragility was $17.5 \%$ and that of pre-fragility, $40.9 \%$. The most frequent fragility clinical criteria were as follows: self-reported fatigue $(42.3 \%)$ and decreased grip strength $(32.8 \%)$. The bivariate analysis showed an association with age, level of education, occupation (class IV and V concerning manual work), comorbidity (class II, asymptomatic disease or asymptomatic disease that requires medication but is under control), polypharmacy, anemia, Parkinson's disease and non-vascular neurological disease. The final prediction model covering the age $(1.08,95 \% \mathrm{Cl}$ : 1.03 to 1.12$)$, higher education level as a preventive variable $(0.21,95 \% \mathrm{Cl}: 0.07$ to 0.62$)$, type II comorbidity $(1108,95 \%$ $\mathrm{Cl}: 1.45$ to 84.38$)$ and polypharmacy $(2.49,95 \% \mathrm{Cl}: 1.24$ to 5.03$)$ predicts the likelihood of fragility syndrome in $75.6 \%$. Conclusions: There is a high prevalence of fragility in the elderly population attending the primary health care service, and a significant association with age, higher education level, type II comorbidity and polypharmacy.
\end{abstract}

Keywords: Adult health; health of the elderly; frail elderly; primary health care (Source: MeSH NLM).

1. Hospital Nacional Almanzor Aguinaga Asenjo, EsSalud. Chiclayo, Perú.

2. Universidad de San Martín de Porres, Filial Norte. Chiclayo, Perú.

a. Médico Geriatra.

b. Médico Epidemiólogo. 
Prevalencia y factores asociados al síndrome de fragilidad en adultos mayores en la consulta de atención primaria de EsSalud, enero-abril 2015. Chiclayo, Perú

\section{INTRODUCCIÓN}

En el mundo actual, la población adulta mayor está envejeciendo, estando en los momentos actuales a representar el $13.5 \%$ de la población mundial de adultos mayores ${ }^{(1)}$. En el Perú, la población de adultos mayores se estima actualmente en $9.7 \%^{(2)}$. En el caso del norte del País, en la Red Asistencial de Lambayeque, la población asegurada aumento 3-4\% y con ello el aumento del grupo de adultos mayores a $15,1 \%{ }^{(3)}$. El crecimiento de la población envejecida está asociada con incremento en la prevalencia de enfermedades crónicas y de los síndromes geriátricos. Entiéndase como síndromes geriátricos aquellos problemas médicos de elevada frecuencia en el adulto mayor y tras su aparición originan un importante deterioro en la calidad de vida de las personas que la padecen y a menudo generan o incrementan la dependencia de otras personas, produciendo un aumento de las necesidades de asistencia sanitaria ${ }^{(4)}$. De todos los síndromes geriátricos, el síndrome de fragilidad tiene una importancia central en la medicina geriátrica.

Aunque no existe un consenso universal con respecto a la definición de fragilidad, en la actualidad son 2 las más aceptadas, una primera sostiene que es un fenotipo con al menos 3 criterios clínicos establecidos de los siguientes: pérdida de peso, autocansancio, fuerza disminuida, velocidad de la marcha lenta y baja actividad física ${ }^{(5)}$; la segunda definición sostiene que es el resultado de una acumulación de deficiencias relacionadas con la salud mediante cuantificación de un extenso rango de signos, discapacidad, medidas de laboratorio y comorbilidad ${ }^{(6)}$. Ambas definiciones coinciden que se trata de un estado de vulnerabilidad al stress que se asocian con eventos adversos ${ }^{(5-7)}$. La fragilidad supone la pérdida excesiva de masa magra corporal, también llamada sarcopenia, como resultado de la interacción entre múltiples factores, tales como: genéticos, procesos de envejecimiento, enfermedades, medio ambiente, estilo de vida, estado de nutrición ${ }^{(8)}$.

El interés del estudio de fragilidad generado en los últimos años, es debido que los sujetos considerados frágiles tienen un riesgo elevado de eventos adversos sobre su salud a corto, medio y largo plazo, es predictor de mortalidad, predictor de discapacidad, hospitalización y de pérdida de movilidad ${ }^{(13,14)}$, entendiéndose que la mayor importancia de identificar un adulto mayor frágil radica en la posibilidad de realizar medidas de prevención primaria y secundaria, con la finalidad de disminuir las tasas de morbimortalidad a largo plazo. Actualmente no existen estudios locales sobre el tema por lo que resulta necesario identificar la prevalencia y factores asociados al síndrome de fragilidad en adultos mayores en la consulta de atención primaria de EsSalud entre enero y abril del 2015.

\section{MATERIALES Y MÉTODOS}

El estudio se desarrolló en el ámbito del primer nivel de atención en el Policlínico Naylamp y Chiclayo Oeste de la Red Asistencial de Lambayeque localizado en el norte del Perú, entre enero y abril del 2015.
Tipo y diseño

Estudio cuantitativo de diseño descriptivo prospectivo y transversal.

\section{Población}

Adultos mayores, según la Organización Mundial de la Salud mayores de 60 años ${ }^{(15)}$, pertenecientes a 2 policlínicos de la Red de Atención Primaria de EsSalud. Ambos con una población adscrita de adultos mayores de 19,486 y 14,848 respectivamente, haciendo un total de 34334.

\section{Muestra}

Se calculó con la calculadora estadística Epidat 3.1 con nivel de confianza del $95 \%$ y un margen de error del $5 \%$, población de 34334, proporción esperada de síndrome de fragilidad de $28 \%{ }^{(11)}$, obteniéndose 308 como minino de muestra y en forma proporcional de ambos policlínicos.

\section{Muestreo}

Se obtuvo el listado de los adultos mayores de los policlínicos, del cual en forma aleatoria se obtuvo 185 del Naylamp y 141 de Chiclayo Oeste. Los pacientes seleccionados fueron ubicados en el Sistema de Gestión Hospitalaria para saber si tenían consulta externa programada para ser captados al momento de su atención, caso contrario fueron ubicados en sus domicilios.

\section{Criterios de selección}

Se incluyeron adultos mayores que caminen independiente o con alguna ayuda mecánica tipo bastón sin apoyo de otra persona, procedan de la ciudad de zona urbana y distritos de Chiclayo y acepten participar en el estudio. Se excluyeron a pacientes que no tengan potencial rehabilitador como los postrados en cama o en sillas de ruedas, con enfermedad neurológica limitante, amputados, ancianos con enfermedades terminales, institucionalización en 3 meses previos, déficit cognitivo severo, enfermedad aguda y con índice geriátrico de comorbilidad clase III y IV (enfermedad sintomática no controlada por terapia y enfermedad que compromete la vida o de gran severidad).

\section{Método}

Se evaluaron los criterios de síndrome de fragilidad, usándose la definición de The Women's Healt and Aging Studies (WHAS), que establece cinco criterios ${ }^{(16)}$ : Pérdida de Peso, definido por más de 4600 kg o más, o igual o mayor del $5 \%$ del peso corporal en el último año; debilidad, medida por la fuerza prensora en el quintil inferior ajustada por el sexo e índice de masa muscular $(<17 \mathrm{~kg}$ para IMC $<=23$; $<=17.3 \mathrm{Kg}$ para IMC entre 23 a 26 ; <=18kg para IMC entre 26 y29; <=21 para IMC >29 ); autocansancio, identificado por 2 preguntas de la escala CES-D ${ }^{(17)}$, que evalúa el esfuerzo que realiza en sus actividades cotidianas; bajo nivel de actividad física, buscándose a los inactivos ${ }^{(18)}$; lentitud, mediante la velocidad para caminar $4.6 \mathrm{~m}$ (PUNTO DE CORTE: $<0.8 \mathrm{~m} / \mathrm{seg})$. Se considera anciano frágil cuando 
presenta 3 criterios, pre-frágil cuando presenta uno o dos criterios y anciano robusto cuando no presenta ninguno $(5,16,19)$, además se recogió información de factores asociados como género, edad, nivel educativo, convivencia, y clase ocupacional previa considerándose en 5 categorías: clase I, si es directivo de administración pública; II, si es directivo de empresa; clase III, empleado de gestión administrativa, trabajadores independientes, supervisores de trabajos manuales, trabajadores de seguridad; IV, trabajadores manuales cualificados (electricistas, mecánicos, conductores, vendedores en tiendas) y $\mathrm{V}$, trabajadores manuales no cualificados (limpieza, peón, no trabaja) ${ }^{(20)}$. Además otras variables como comorbilidad evaluado con el índice geriátrico de comorbilidad considerando si es Clase I o II, según tenga ausencia de enfermedad o enfermedad asintomática; o enfermedad asintomática que requiere medicación y está bajo control, respectivamente ${ }^{(21)}$; polifarmacia si toma más de 3 medicamentos ${ }^{(22)}$.

\section{Análisis de datos}

Para el registro y manejo de datos se utilizó el programa Stata versión 13, para los datos demográficos se utilizó porcentaje, medidas de tendencia central como media y desviación estándar, para buscar asociación RP (razón de prevalencia) con IC 95\% y para inferencia estadística chi cuadrado. Las variables que obtuvieron un $\mathrm{p}<0.05$ se incorporaron a un modelo de lineales generalizado (GLM) con análisis binomial. Para la obtención del modelo predictivo se ingresaron las variables con valores $p<0,10$ en el análisis bivariado. En el modelo final solo se incluyeron a las variables $p<0,05$ y se calculó el coeficiente de determinación del modelo. Para la elaboración de tablas y gráficos se usó Excel de Windows 2013.

\section{Aspectos éticos}

El estudio fue revisado y aprobado por el comité de investigación del Hospital Nacional Almanzor Aguinaga Asenjo, guardando la confidencialidad de la información recolectada.

\section{RESULTADOS}

En la figura 1 se puede observar que la prevalencia del síndrome de fragilidad fue de $17.5 \%$ (57 casos), prefragilidad $40.9 \%$ (133 casos) y robustos $41.6 \%$ (136 casos).

Del total de adultos mayores $42.3 \%$ (138) correspondió al grupo etario entre 71 y 80 años, seguido con $30.7 \%$ (100) del grupo etario de 60 a 70 años .La población femenina fue la más frecuente con $55.2 \%$, en cuanto convivencia, las personas que vivían con cónyuge, y otro grupo sólo con hijos presentaron mayor prevalencia, $45.4 \%$ (148) y 43.9\% (143) respectivamente; en la ocupación, la clase IV y $\mathrm{V}$ representó la de mayor frecuencia; en cuanto el nivel educativo, $39.6 \%$ presentaron primaria, $31.6 \%$ secundaria.

Sobre la frecuencia de criterios clínicos del síndrome de fragilidad, el cansancio referido y la fuerza disminuida se reportaron como los más frecuentes con $42.3 \%$ y $32.8 \%$ respectivamente, seguido de marcha lenta $19,3 \%$ ), peso disminuido $(17,2 \%)$ y baja actividad física $(8,9 \%)$.



Figura 1. Prevalencia del síndrome de fragilidad en adultos mayores en la consulta de atención primaria de EsSalud. Enero-abril, 2015. Chiclayo, Perú

De los 57 adultos mayores con fragilidad, se encontró mayor frecuencia en los grupos etario 81 a 90 años con $27,3 \%$ (21 casos) y $>91$ años $63.6 \%$ (7 casos); de género femenino $18,9 \%$ (34 casos), respecto al grado de instrucción, la mayor frecuencia son los analfabetos con 42,9\% (6 casos). Dentro de la convivencia, el no vivir con resto de familia $21,4 \%$ (3 casos). Y la ocupación principalmente fueron trabajadores manuales cualificados o no cualificados con $50 \%$ (45) (Tabla 1).

La mayoría de adultos mayores con síndrome de fragilidad pertenece a la clase de comorbilidad II, esto es un 17,2\% (56 casos) que significa comorbilidad con 1 o más enfermedades crónicas asintomáticas que requiere medicación pero que 
Prevalencia y factores asociados al síndrome de fragilidad en adultos mayores en la consulta de atención primaria de EsSalud, enero-abril 2015. Chiclayo, Perú

está bajo control. Así mismo, existe un 6,1\%(20 casos) de adultos mayores con síndrome de fragilidad que tienen polifarmacia. Dentro de las comorbilidades relacionadas al síndrome de fragilidad, la hipertensión arterial presentó la mayor frecuencia en los adultos mayores frágiles, con $64.91 \%$ ( 37 casos), seguida de $45.61 \%$ ( 26 casos) que presentaron enfermedad músculo esquelética, y el $19.30 \%$ (11 casos) parkinsonismo y enfermedad neurológica no vascular (Tabla 1).

Tabla 1. Síndrome de fragilidad según factores sociodemográficos, comorbilidad y polifarmacia en adultos mayores en consulta de dos centros de atención primaria de EsSalud. Enero-abril, 2015. Chiclayo, Perú.

\begin{tabular}{|c|c|c|c|c|c|c|}
\hline \multirow[t]{2}{*}{ Factores } & Total & Fragilidad & \multicolumn{4}{|l|}{$\mathbf{N}^{\circ}$ Fragilidad } \\
\hline & $\mathrm{n}$ & $\%$ & $n=57$ & $\%$ & $n=269$ & $\%$ \\
\hline \multicolumn{7}{|l|}{ Género } \\
\hline Masculino & 146 & $44.8 \%$ & 23 & $15.8 \%$ & 123 & $84.2 \%$ \\
\hline Femenino & 180 & $55.2 \%$ & 34 & $18.9 \%$ & 146 & $81.1 \%$ \\
\hline Edad & & & & & 0 & \\
\hline 60-70 años & 100 & $30.7 \%$ & 8 & $8.0 \%$ & 92 & $92.0 \%$ \\
\hline 71-80 años & 138 & $42.3 \%$ & 21 & $15.2 \%$ & 117 & $84.8 \%$ \\
\hline $81-90$ años & 77 & $23.6 \%$ & 21 & $27.3 \%$ & 56 & $72.7 \%$ \\
\hline$>91$ años & 11 & $3.4 \%$ & 7 & $63.6 \%$ & 4 & $36.4 \%$ \\
\hline \multicolumn{7}{|l|}{ Grado de instrucción } \\
\hline Analfabeto & 14 & $4.3 \%$ & 6 & $42.9 \%$ & 8 & $57.1 \%$ \\
\hline Primaria & 129 & $39.6 \%$ & 28 & $21.7 \%$ & 101 & $78.3 \%$ \\
\hline Secundaria & 103 & $31.6 \%$ & 19 & $18.4 \%$ & 84 & $81.6 \%$ \\
\hline Superior & 80 & $24.5 \%$ & 4 & $5.0 \%$ & 76 & $95.0 \%$ \\
\hline \multicolumn{7}{|l|}{ Convivencia } \\
\hline Vive solo & 21 & $6.4 \%$ & 2 & $9.5 \%$ & 19 & $90.5 \%$ \\
\hline Con cónyuge & 148 & $45.4 \%$ & 22 & $14.9 \%$ & 126 & $85.1 \%$ \\
\hline Con hijos & 143 & $43.9 \%$ & 30 & $21.0 \%$ & 113 & $79.0 \%$ \\
\hline Con resto de la familia & 14 & $4.3 \%$ & 3 & $21.4 \%$ & 11 & $78.6 \%$ \\
\hline \multicolumn{7}{|l|}{ Ocupación } \\
\hline Clase I & 23 & $7.1 \%$ & 1 & $4.3 \%$ & 22 & $95.7 \%$ \\
\hline Clase II & 43 & $13.2 \%$ & 1 & $2.3 \%$ & 42 & $97.7 \%$ \\
\hline Clase III & 80 & $24.5 \%$ & 10 & $12.5 \%$ & 70 & $87.5 \%$ \\
\hline Clase IV & 92 & $28.2 \%$ & 22 & $23.9 \%$ & 70 & $76.1 \%$ \\
\hline Clase V & 88 & $27.0 \%$ & 23 & $26.1 \%$ & 65 & $73.9 \%$ \\
\hline \multicolumn{7}{|l|}{ Comorbilidad } \\
\hline Clase I & 54 & $16.6 \%$ & 1 & $1.9 \%$ & 53 & $98.1 \%$ \\
\hline Clase II & 272 & $83.4 \%$ & 56 & $20.6 \%$ & 216 & $79.4 \%$ \\
\hline \multicolumn{7}{|l|}{ Polifarmacia } \\
\hline sí & 64 & $19.6 \%$ & 20 & $31.3 \%$ & 44 & $68.8 \%$ \\
\hline NO & 262 & $80.4 \%$ & 37 & $14.1 \%$ & 225 & $85.9 \%$ \\
\hline
\end{tabular}


Al realizar el análisis de asociación de los factores sociodemográficos, se observa que por cada año aumenta 6\% (IC95\%: 1,04-1,08) las probabilidades, así el estar entre 81-90 años aumenta 3,4 veces (IC95\%: 1.59-7.27) y tener >91 años aumenta 7,9 veces (IC95\%: 3,57-17,71) de tener síndrome de fragilidad, así también el grado de instrucción secundaria disminuye 57\% (IC95\%: 11\%-80\%) e instrucción superior 89\% (IC95\%: 64\%-97\%) las probabilidades de tener síndrome de fragilidad.

La presencia de comorbilidad clase Il (1 o más enfermedades crónicas asintomáticos que requieren medicación, y que esté bajo control) aumenta 11,11 veces la probabilidad de tener síndrome de fragilidad, Con relación a polifarmacia, la probabilidad es 2.21 veces (IC95\%: 1.38-3.54) de tener síndrome de fragilidad que los que no tenían polifarmacia. Sólo el parkinsonismo y la enfermedad neurológica no vascular constituyó una enfermedad, que quienes la padecen tenían 81\% (IC95\%: 1.03-3.18) más de probabilidad de hacer síndrome de fragilidad. Para el análisis multivariado se incluyeron las variables con $\mathrm{p}<0,10$ para realizar el modelo de predicción, por lo que se incluyó a grado de instrucción primaria, ocupación clase IV y V, anemia, comorbilidad clase II (Tabla 2).

Tabla 2. Factores sociodemográficos y clínicos asociados al síndrome de fragilidad en adultos mayores en consulta de dos centros de Atención primaria de EsSalud. Enero-abril, 2015. Chiclayo, Perú.

\begin{tabular}{|c|c|c|c|c|}
\hline \multirow[t]{2}{*}{ Factores } & \multirow[t]{2}{*}{ RP } & \multicolumn{2}{|c|}{ IC $95 \%$} & \multirow[t]{2}{*}{$\mathbf{p}$} \\
\hline & & LI & LS & \\
\hline \multicolumn{5}{|l|}{ Sociodemográficos } \\
\hline Edad (años) & 1,06 & 1,04 & 1,08 & 0,001 \\
\hline $81-90$ años & 3,40 & 1,59 & 7,27 & 0,002 \\
\hline$>91$ años & 7,95 & 3,57 & 17,71 & $<0,001$ \\
\hline \multicolumn{5}{|l|}{ Grado de instrucción } \\
\hline Primaria & 0,50 & 0,25 & 1,00 & 0,053 \\
\hline Secundaria & 0,43 & 0,20 & 0,89 & 0,023 \\
\hline Superior & 0,11 & 0,03 & 0,36 & $<0,001$ \\
\hline \multicolumn{5}{|l|}{ Ocupación } \\
\hline Clase IV & 5,5 & 0,78 & 38,70 & 0,08 \\
\hline Clase V & 6,01 & 0,85 & 42,20 & 0,07 \\
\hline \multicolumn{5}{|l|}{ Clínicos } \\
\hline Comorbilidad (clase II) & 11,11 & 1,57 & 78,58 & 0,016 \\
\hline Polifarmacia & 2,21 & 1.38 & 3.54 & $<0,001$ \\
\hline Anemia & 2,16 & 0,95 & 4,90 & 0,06 \\
\hline $\begin{array}{l}\text { Parkinson y enfermedad Neurológicas no } \\
\text { vascular }\end{array}$ & 1,81 & 1,03 & 3,18 & 0,03 \\
\hline \multicolumn{5}{|l|}{ Severidad de la Enfermedad } \\
\hline Asintomática & 7.50 & 0,85 & 66,12 & 0,07 \\
\hline Asintomática con medicación bajo control & 6,69 & 0,95 & 46,92 & 0,056 \\
\hline
\end{tabular}

El modelo de predicción de los factores relacionados al síndrome de fragilidad, se puede apreciar que la edad (años), grado de instrucción superior, comorbilidad tipo II y polifarmacia, son factores que podrían predecir la probabilidad de tener síndrome de fragilidad en un 75,6\%\% (Tabla 3). 
Prevalencia y factores asociados al síndrome de fragilidad en adultos mayores en la consulta de atención primaria de EsSalud, enero-abril 2015. Chiclayo, Perú

Tabla 3. Modelo de predicción al síndrome de fragilidad en adultos mayores en consulta de dos centros de atención primaria de EsSalud. Enero-abril, 2015. Chiclayo, Perú.

\begin{tabular}{lrrrr} 
Factores & PR ajustados* & \multicolumn{2}{c}{ IC 95\% } & P \\
Edad (años) & 1,08 & 1,03 & 1,12 & 0,01 \\
Grado Instrucción & 0,21 & 0,07 & 0,62 & 0,005 \\
(Superior) & 11,08 & 1,45 & 84,38 & 0,02 \\
Comorbilidad (tipo II) & 2,49 & 1,24 & 5,03 & 0,01 \\
Polifarmacia & & & &
\end{tabular}

* Calculado con Modelo Lineales Generalizados (GLM)

El modelo tiene una predicción de 75,6\%

\section{DISCUSIÓN}

Los estudios sobre fragilidad en el anciano, se han realizado desde que Fried en el año 2001, establece los criterios para su identificación, en aquel entonces encontró que $6.9 \%$ eran frágiles ${ }^{(5)}$. En América, un estudio con diseño similar realizado en Canadá en el 2005, halló $22.7 \%^{(19)}$. Algunos estudios de prevalencia en países latinoamericanos arrojan: 15.7 en México ${ }^{(23)}$, 9,1\% en Brasil ${ }^{(24)}, 12,1 \%$ en Colombia ${ }^{(25)}$. En nuestro país, se han realizado 2 estudios en Lima, una realizada por Varela encontrando una prevalencia de $7.7 \%{ }^{(11)}$ y Runzer un porcentaje mucho mayor $27.8 \%^{(12)}$, la prevalencia encontrada en nuestro estudio fue de $17.5 \%$ un hallazgo intermedio entre lo encontrado por los autores anteriores, probablemente en estos dos últimos casos la prevalencia fue mayor por ser población que acude a establecimientos de salud.

Otras posibles explicaciones a esta variabilidad de la prevalencia encontrada puede deberse a la diferencia de características socioculturales de las poblaciones evaluadas, metodología del tipo de investigación y el impacto de la frecuencia de enfermedades crónicas cada vez mayor conforme pasan los años, ya que las prevalencia de fragilidad, en los últimos 5 años, no ha bajado del 10\%, y algunos estudios incluso, superan el $20 \%$ como la de Song $X^{(19)}$ y Runzer $F^{(12)}$. Otro dato importante encontrado, es la prevalencia de pre fragilidad: $40.80 \%$, mientras que Runzer ${ }^{(12)}$, encuentra $47.3 \%$ y Varela $64.6 \%{ }^{(11)}$, Sánchez ${ }^{(23)}$ en México encuentra $33.3 \%$ y Gómez ${ }^{(25)}$ en Colombia encuentra 53\%. Esto es importante, teniendo en cuenta que probablemente si a éstos pacientes no se les realiza un programa de intervención, en los próximos tres años tendrán el doble de riesgo a pasar a la condición de frágil (26) con todas las consecuencias que esto ocasiona.

Con relación a los criterios de frecuencia de los criterios de fragilidad, se halló principalmente agotamiento (o cansancio autoreferido) y debilidad (fuerza disminuida), a diferencia de Varela reporta a debilidad y pérdida de peso ${ }^{(11)}$, un estudio realizado en España ${ }^{(27)}$ refiere disminución de fuerza y marcha lenta, esta variabilidad de hallazgos podría estar relacionado con factores relacionadas a comorbilidad y medicación, y según un estudio local se tienen de 3-6 fármacos prescritos ${ }^{(28)}$, sin embargo el hallazgo común en los 3 estudios, fue la alta frecuencia de fuerza disminuida.

Entre los hallazgos resalta que por cada año aumenta $8 \%$ la presencia del síndrome de fragilidad, aunque no se encontró asociación con el género, respecto a la edad, los hallazgos coinciden con los trabajos de Varela ${ }^{(11)}$, Runzer ${ }^{(12)}$, Gómez ${ }^{(25)}$, Moreira ${ }^{(24)}$, Sánchez ${ }^{(23)}$, Song (19) Castell (9), no así con el género probablemente por la distribución que tiene la pirámide poblacional de la población asegurada con tendencia al sexo femenino ${ }^{(3)}$. Los estudios de Gómez y Moreira a igual que de Castell coinciden con nuestros hallazgos refiriendo que el nivel superior disminuye las probabilidades de la presencia de síndrome de fragilidad ${ }^{(24,25)}$, probablemente por su asociación al deterioro cognitivo ${ }^{(29)}$. Con relación a la convivencia tenemos alto porcentaje de pacientes frágiles que no tiene pareja y viven con uno o varios hijos, Runzer realizó su estudio en familiares de veteranos de la marina peruana ${ }^{(12)}$, encontró alto frecuencia de casados en estado de fragilidad, y Sánchez-García en México (23) halló alta frecuencia de frágiles en pacientes que viven solos; estos hallazgos deberían ser bien estudiados la definición de la variable, dado que se puede tener estado civil casado, pero está viviendo solo o con sus hijos. Otra variable estudiada fue la ocupación previa, aunque inicialmente mostraba asociación con el trabajo manual similar a lo reportado por Sánchez-García ${ }^{(23)}$, pero en el modelo final no lo incluye, probablemente se presente porque estudios refieren que el SF está asociado a la adultos mayores que no realizan ninguna actividad productiva y el dato recogido en base a la ocupación previa ${ }^{(29)}$.

La comorbilidad es un factor asociado importante, sólo bastaría ver que el $83.4 \%$ informa estar en clase II (1 o más enfermedades crónicas asintomáticas que requiere 
medicación pero que está bajo control), siendo similar a estudios en Río de Janeiro ${ }^{(24)}$, México ${ }^{(23)}$, España ${ }^{(9)}$, si bien la frecuencia de enfermedades crónicas difiere en cada país, en el Perú la hipertensión arterial y las enfermedades músculo-esqueléticas son frecuentes en ancianos frágiles, también lo son en no frágiles, sin embargo el parkinsonismo y la enfermedad neurológica no vascular en el análisis bivariado presentó asociación, quizás por la presencia de deterioro cognitivo leve es más frecuente en el sexo femenino, en personas de mayor edad realizado en la misma población ${ }^{(30)}$, además hallazgo similar en un estudio en España ${ }^{(31)}$; mientras que la polifarmacia además de tener una alta frecuencia que otros estudios locales también lo refieren ${ }^{(28)}$, también estuvo asociado al igual que refiere el estudio de Sánchez $\mathrm{P}^{(31)}$ en España. No hemos encontrando estudios en América latina que mencionen algún modelo predictivo de la presencia de Síndrome de Fragilidad, pero encontramos que la edad, grado de instrucción superior como variable preventiva, comorbilidad y polifarmacia predicen la probabilidad de tener síndrome de fragilidad en un $75,6 \%$, aunque una limitación del estudio es el diseño trasversal que no permite una relación causa efecto.

En conclusión, este estudio muestra una alta prevalencia de fragilidad en la población de adultos mayores que acuden a la consulta de atención primaria de EsSalud. Chiclayo, Perú con un porcentaje considerable de ancianos prefrágiles y con una asociación significativa con la edad, grado de instrucción superior, comorbilidad y polifarmacia.

Es necesaria la inclusión de colocar en práctica la evaluación de los criterios de identificar el síndrome de fragilidad en la consulta de atención primaria para hacer un diagnóstico precoz y posteriormente establecer programas de intervención que modifique los eventos adversos asociados. En nuestro medio, la población potencialmente beneficiaria del cribado de fragilidad serían los adultos mayores con los factores de riesgo identificados.

\section{REFERENCIAS BIBLIOGRÁFICAS}

1. Galbán PA, Soberats FJS, Navarro AMD-C, García MC, Oliva T. Envejecimiento poblacional y fragilidad en el adulto mayor. Rev Cuba Salud Pública [Internet]. 2007 [acceso mayo del 2017];33(1). Disponible en: http://scielo.sld.cu/scielo. php?script=sci_arttext\&pid=S0864-34662007000100010

2. INEI. Población y vivienda. [Internet]. [acceso el 31 de julio de 2017]. Disponible en: https://www.inei.gob.pe/estadisticas/ indice-tematico/poblacion-y-vivienda/

3. Díaz-Vélez C, Ñanfuñay-Silva R, Yangua-Jaramillo A, ChumanCarmen A. Capacidad de atención de Hospitales (HNAAA, H. Luis Heysen Inchaustegui, H. Naylamp). Lambayeque-Perú: Essalud; 2015. Documento de trabajo: 14

4. Gómez A-E. Grandes síndromes geriátricos: descripción. Farm Prof. 2005;19(6):70-4.

5. Fried L, Tangen C. Walston J, Newman A, Hirsch C, Gottdiener $\mathrm{J}$, et al. Frailty in older adults evidence for a phenotype. J Gerontol A Biol Sci Med Sci. 2001;56(3):M146-M57.

6. Rockwood K, Mitnitski A. Frailty in relation to the accumulation of deficits. J Gerontol A Biol Sci Med Sci. 2007;62(7):722-7.

7. Díaz de León E, Tamez H, Gutiérrez H, Cedillo J, Torres G. Frailty and its association with mortality, hospitalization and functional dependency among 60 year and older Mexican people. Med Clin Barc. 2012;138(11):468-74.

8. Heuberger R. The frailty syndrome: a comprehensive review. J Nutr Gerontol Geriatr. 2011;30(4):315-68.

9. Castella-Alcalá $M$, Melgar A, Viñals $R$, Hoyos-Alonso $M$. Consideraciones sobre los estudios de prevalencia de fragilidad en el mayor en España. Aten Primaria. 2012;44(5):295-6.

10. Alvarado B, Zunzunegui $M$, Béland $F$, Bamvita J. Life course social and health conditions linked to frailty in Latin American older men and women. J Gerontol A Biol Sci Med Sci. 2008;63(12):1399-406.

11. Varela-Pinedo L, Ortiz-Saavedra P, Chávez-Jimeno H. Síndrome de fragilidad en adultos mayores de la comunidad de Lima Metropolitana. Rev Soc Peru Med Interna. 2008;21(1):11-5.

12. Runzer-Colmenares F, Samper-Ternent R, Al Snih S, Ottenbacher K, Parodi J, Wong R. Prevalence and factors associated with frailty among Peruvian older adults. Arch Gerontol Geriatr. 2014;58(1):69-73.

13. Abizanda P, López-Torres H, Romero L, Sánchez P, García I, Esquinas J. Normal data of functional assessment tools of the elderly in Spain: the FRADEA study. Atencion Primaria. 2012;44(3):162-71.

14. Ávila-Funes J, Helmer C, Amieva H, Barberger-Gateau P, Le Goff $M$, Ritchie K, et al. Frailty among community-dwelling elderly people in France: the three-city study. J Gerontol A Biol Sci Med Sci. 2008;63(10):1089-96.

15. OMS | Envejecimiento [Internet]. Who.int. [actualizada el 2017; acceso el 27 de diciembre de 2016]. Disponible en: http://www.who.int/topics/ageing/es/

16. Van Kan G, Rolland Y, Bergman H, Morley J, Kritchevsky S, Vellas B, et al. The IANA Task Force on frailty assessment of older people in clinical practice. J Nutr Health Aging. 2008;12(1):29-37.

17. Ruiz-Grosso P, Loret C, Vega-Dienstmaier J, Arevalo J, Chavez K, Vilela A, et al. Validation of the spanish Center for epidemiological studies depression an zung self Rating Depresion scales: a comparative validation study. Plos One. 2012;7(10): e45413.

18. Washburn R, Smith K, Jette A, Janney C. The physical Activity scale for the Elderly (PASE): Development and evaluation. $J$ Clin Epidemiol.1993;46(2):153-62

19. Song X, Mitnitski A, Rockwood K. Prevalence and 10-Year Outcomes of Frailty in Older Adults in Relation to Deficit Accumulation. J Am Geriatr Soc. 2010;58(4):681-7.

20. Domingo-Salvany A, Regidor E, Alonso J, Álvarez-Dardel C, Borrell C, Doz F, et al. Una propuesta de medida de la clase social. Aten Primaria. 2000;25(5):350-63.

21 Rosas-Carrasco O, González-Flores E, Brito-Carrera A, VázquezValdez 0 , Peschard-Sáenz E, Gutiérrez-Robledo L, et al. Evaluación de la comorbilidad en el adulto mayor. Rev Médica Inst Mex Seguro Soc. 2011;49(2):153-62.

22. Millan J. Gerontología y geriatría: valoración e intervención. Madrid. Editorial Médica Panamericana; 2011.

23. Sánchez-García S, Sánchez-Arenas R, García-Peña C, RosasCarrasco O, Ávila-Funes J, Ruiz-Arregui L, et al. Frailty among community-dwelling elderly Mexican people: Prevalence and association with sociodemographic characteristics, health state and the use of health services. Geriatr Gerontol Int. 2014;14(2):395-402.

24. Moreira V, Lourenco R. Prevalence and factors associated with frailty in an older population from the city of Rio de Janeiro, Brazil: the FIBRA-RJ Study. Clinics. 2013;68(7):979-85.

25. Gomez F, Curcio C, Henao G. Fragilidad en ancianos 
Prevalencia y factores asociados al síndrome de fragilidad en adultos mayores en la consulta de atención primaria de EsSalud, enero-abril 2015. Chiclayo, Perú

colombianos. Rev Medica Sanitas. 2012;15(4):8-16.

26. Toscano M. Sindrome de Fragilidad. [citado el 28 de diciembre de 2016]; Disponible en: http://dsp.facmed.unam.mx/ censenanza/spivsa/antol\%202\%20anciano/2parte2013/II_ sindrome.pdf

27. Abizanda P, López-Torres J, Romero L, Lopéz $M$, Sánchez $P$, Atienzar $\mathrm{P}$, et al. Fragilidad y dependencia en Albacete (estudio FRADEA): razonamiento, diseño y metodología. Rev Esp Geriatría Gerontol. 2011;46(2):81-8.

28. Díaz-Nolazco $M$, Roque-Alvarado J, Corimanya-Capitán $B$, Remón-Malca M, Puescas-Sánchez P, Díaz-Vélez C. Calidad de prescripción de medicamentos en adultos mayores. EsSalud-2011. Rev Cuerpo Med HNAAA. 2012;5(4):12-7.

29. Villacís A, Aguilar H. Prevalencia del síndrome de fragilidad y factores asociados en adultos mayores de la Parroquia San Joaquín del Cantón Cuenca, 2012. [Tesis bachiller]. Cuenca: Universidad de Cuenca; 2014.

30. Chávez-Romero L, Núñez-López I, Díaz-Vélez C, Poma-Ortiz J. Tamizaje de deterioro cognitivo leve en adultos mayores con enfermedad cardiovascular en un Hospital Nacional de Chiclayo, Perú. Rev Méd Risaralda. 2014;20(1):14-9.

31. Sánchez Jurado PM. Prevalencia y atributos de la fragilidad en una cohorte española mayor de 70 años. [Tesis doctoral]. Albacete: Universidad de Castilla-La Mancha; 2013.
Fuentes de financiamiento:

Este artículo ha sido financiado por los autores.

Conflictos de interés:

Los autores declaran no tener ningún conflicto de interés.

\section{Correspondencia:}

Milton Carlos Gonzáles Mechán

Dirección: Chiclayo, Perú.

Teléfono: 979549456

Correo electrónico: mcgonzales8@hotmail.com

Recibido: 30 de febrero de 2017

Evaluado: 06 de abril de 2017

Aprobado: 26 de junio de 2017

( ) La revista. Publicado por Universidad de San Martín de Porres, Perú. (cc) Br Licencia de Creative Commons Artículo en acceso abierto bajo términos de Licencia Creative Commons Atribución 4.0 Internacional. (http://creativecommons.org/licenses/by/4.0/) 